Cómo citar este artículo:

G.A. Jiménez-Tovar, and M. Parra-Pinilla, "Lixiviación de cobre contenido en tarjetas de computador PCB para la extracción de metales preciosos”, Rev. Ing. Investig. Desarro., vol. 18 (2), pp. 15-21, Julio. 2018.

\title{
LIXIVIACIÓN DE COBRE CONTENIDO EN TARJETAS DE COMPUTADOR PCB PARA LA EXTRACCIÓN DE METALES PRECIOSOS
}

\section{Lixiviation of copper content in pcb computer cards for the extraction of precious metals}

\author{
Ginna Alejandra Jiménez Tovar ${ }^{1}$, Mario Parra Pinilla ${ }^{1}$ \\ ${ }^{1}$ MSc. Universidad Pedagógica y Tecnológica de Colombia (Tunja, Colombia). ginna.jimenez@uptc.edu.co
}

(Recibido noviembre 6 de 2017 y aceptado junio 25 de 2018)

\begin{abstract}
Resumen
La recuperación de oro y plata desde residuos electrónicos es una alternativa amigable con el medio ambiente que puede complementar la producción industrial de ellos, usualmente obtenidos a partir de menas naturales y también para promover la reducción de dichos residuos. Como paso previo a la recuperación de estos metales es necesario extraer el cobre contenido en ellos y lograr así una mayor recuperación. En el presente estudio se analizó la extracción de cobre a partir de tarjetas de computador PCB. Se inició con una caracterización de los metales de interés para luego estudiar su comportamiento en el proceso de disolución. A continuación, se realizó un proceso de lixiviación con ácidos inorgánicos a condiciones ambientales durante seis horas y agitación mecánica de 300 RPM. Al finalizar, se eligió la lixiviación con mayor porcentaje de cobre extraído y se hizo una caracterización de los residuos donde se muestra que el contenido de cobre disminuyó desde 27,09\% hasta 1.48\%. Así mismo, la concentración de oro varió desde 131,29 g/ton hasta 345,9g/ ton y la de la plata desde $310 \mathrm{~g} /$ ton hasta $864,8 \mathrm{~g} / \mathrm{ton}$. Para comprobar el proceso de limpieza de cobre se llevo a cabo un proceso de lixiviación con soluciones de tiourea obteniéndose resultados de $60,9 \%$ y $96,06 \%$ para plata y oro, respectivamente.
\end{abstract}

Palabras clave: ácidos inorgánicos, cobre, limpieza, lixiviación, PCB.

\begin{abstract}
The recovery of gold and silver from electronic waste is a friendly alternative to the environment that can complement the industrial production of them, usually obtained from natural ores and also to promote the reduction of such waste1. As a step prior to the recovery of these metals it is necessary to extract the copper contained in them and thus achieve a greater recovery. In the present study, the extraction2 of copper was analyzed from PCB computer cards. It began with a characterization of the metals of interest to then study their behavior in the dissolution process. Next, a process of leaching with inorganic acids was carried out at ambient conditions for six hours and mechanical agitation of 300 RPM. At the end, leaching was chosen with the highest percentage of copper extracted and a characterization of the waste was made showing that the copper content decreased from $27.09 \%$ to $1.48 \%$. Likewise, the concentration of gold varied from $131.29 \mathrm{~g} /$ ton to $345.9 \mathrm{~g} /$ ton and that of silver from $310 \mathrm{~g} /$ ton to $864.8 \mathrm{~g} / \mathrm{ton}$. To verify the copper cleaning process, a leaching process was carried out with thiourea solutions, obtaining results of $74.79 \%$ and $64.8 \%$ for silver and gold, respectively.
\end{abstract}

Key words: inorganic acid, copper, cleaning, leaching, PCB.

\section{INTRODUCCIÓN}

El desarrollo tecnológico en los equipos eléctricos y electrónicos que se ha generado por la evolución e innovación de los últimos años está ayudando al impulso de nuevas tecnologías para la industria. [1]
Sin embargo, se ha aumentado la cantidad de residuos electrónicos en hogares y empresas al punto que, en Colombia hasta el año 2013, se habían generado más de 143.000 toneladas, y para el año 2016 ascendió a 275.000 toneladas [2 - 3]. Este hecho ha causado inconvenientes de contaminación ambiental debido a 
que los elementos químicos que contienen, poliméricos, cerámicos y metálicos, especialmente los metales pesados, como el plomo y el cadmio, son perjudiciales para el planeta y en consecuencia para la salud de los seres humanos. [4]

La creciente recolección y su consecuente acumulación ha planteado la necesidad de crear procesos alternativos para intentar la recuperación de metales comunes $(\mathrm{Cu}, \mathrm{Fe}$, $\mathrm{Ni})$, metales preciosos ( $\mathrm{Au}, \mathrm{Ag}$ ) y otros elementos químicos de interés tecnológico. Dichas metodologías, enmarcadas dentro de una metalurgia secundaria, podrían constituirse en un proceso alternativo para complementar el uso de las menas naturales para la recuperación de tales metales. Además, se realiza un mejor tratamiento de los materiales antropogénicos evitando que lleguen a vertederos donde se convierten en un problema ambiental. [5-8]

En los residuos electrónicos PCB (Printed Circuit Board) el metal que se presenta en mayor proporción es el cobre, el cual aparece en cantidades cercanas al $25 \%$ de los valores metálicos totales. En algunos estudios de recuperación de cobre, en procesos de lixiviación con ácidos inorgánicos, se han obtenido resultados hasta del 98\% de extracción. [9]

Por otra parte, al comparar las leyes de oro y plata en los minerales tradicionales con los contenidos generalmente mostrados por los residuos PCB, la diferencia es bastante notoria. Tal hecho motiva para que se intente su recuperación desde tales materiales. Sin embargo, para lograr su mejor extracción, se hace necesario eliminar los metales comunes ya descritos y evitar así el consumo extra de reactivos que se generaría por la presencia de ellos. [911]

El objetivo del presente estudio consiste entonces en limpiar los residuos electrónicos PCB, extrayendo el cobre presente en ellos, mediante disoluciones o lixiviaciones realizadas a condiciones ambientales con ácidos inorgánicos utilizados como agentes disolventes. A partir de los resultados de este trabajo, se busca establecer las condiciones más recomendables para efectuar pretratamientos que permitan la posterior recuperación de metales preciosos.

\section{Análisis Termodinámico}

La mayoría de las reacciones en hidrometalurgia pueden escribirse para que muestren la extensión en que ellas ocurren o pueden ocurrir, dependiendo del pH de las soluciones y el potencial oxido-reducción. Al graficar en un plano de coordenadas estas variables se obtiene el diagrama de áreas predominantes o Pourbaix, que permite realizar los análisis adecuados para establecer si una reacción ocurrirá o no y en qué condiciones. [12]

En la figura. 1, se muestra el diagrama para el cobre, donde se presenta el análisis termodinámico para cada una de las especies. La información termodinámica se obtuvo de la base de datos NIST 46, versión 8.0 (NIST, 2004) y se integró en la suite de software MEDUSA (Diagramas de equilibrio usando algoritmos simples).

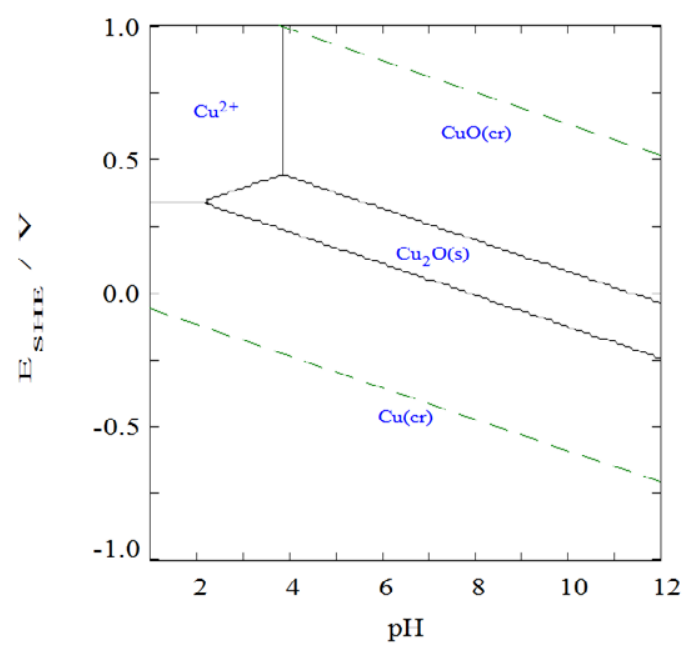

Figura 1. Diagrama de predominancia $\mathrm{Eh} / \mathrm{pH}$ a $25^{\circ} \mathrm{C}$ del sistema Cu- $\mathrm{H}_{2} \mathrm{O}[13]$

Al analizar el diagrama de predominancia para el cobre, el proceso de lixiviación se podría realizar en un intervalo de $\mathrm{pH} 0$ a 4.0 y el metal seria soluble en potenciales mayores a $0.3 \mathrm{~V}$.

\section{MATERIALES Y MÉTODOS}

\subsection{Preparación del Material}

Una muestra de $1400 \mathrm{~g}$ de tarjetas de diferentes tipos de computadores, computadores portátiles y de escritorio, fue sometida a reducción de tamaño utilizando un molino de martillos de alto impacto que garantizara su rompimiento dada la alta cohesión mostrada por esta clase de material, figura 2. Finalizada esta operación, se realizó un análisis de distribución de tamaño de partícula de los diferentes materiales obtenidos, los cuales se muestran en la figura 3. 


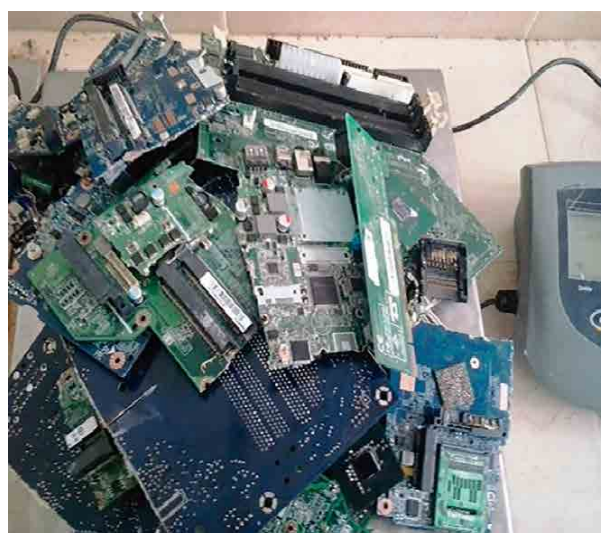

a)

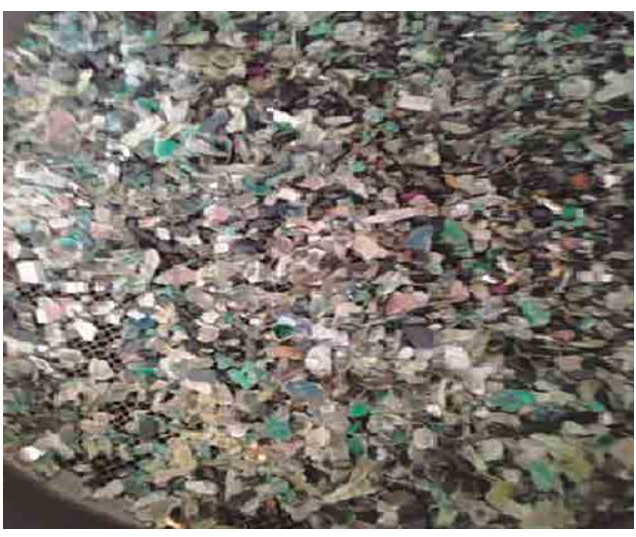

b)

Figura 2. Tarjetas de computador PCB. a) sin triturar b) trituradas

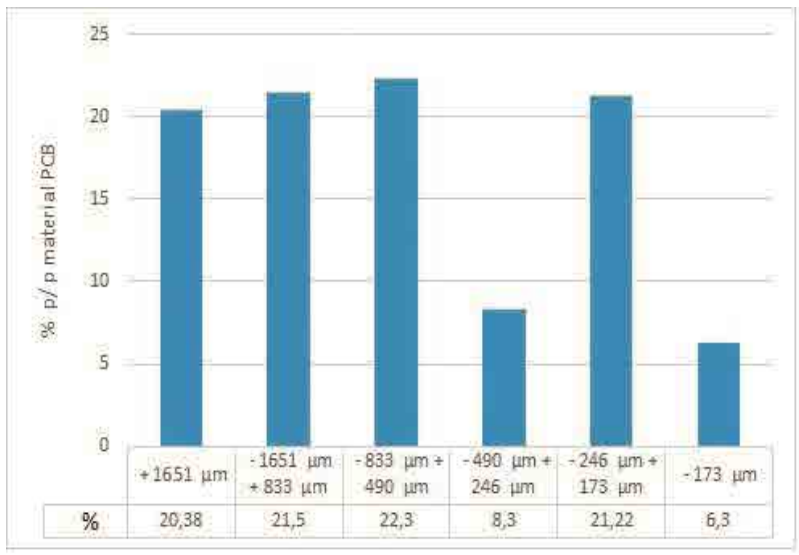

Figura 3. Distribución de tamaño de partícula

Dados estos resultados se seleccionó como muestra para trabajo, el tamaño entre los rangos $-833+490 \mu \mathrm{m}$ por ser el tamaño en que se acumula mayor porcentaje en peso de residuo.

Las soluciones para lixiviación o disolución de los valores metálicos contenidos en las muestras de trabajo fueron preparadas a partir de ácidos grado analítico (ácido clorhídrico 37\% J.T Baker; ácido sulfúrico 97\% Honeywell y ácido nítrico 65\% Emsure). Para la lixiviación de los metales preciosos oro y plata se utilizó tiourea concentrada de grado analítico marca Panreac y peróxido de hidrógeno concentrado 30\% J.T Baker Las diluciones necesarias para ajustar las concentraciones empleada durante los experimentos fueron preparadas con agua destilada y desionizada.

\subsection{Digestión}

Para la cuantificación de los valores metálicos de interés, se hicieron digestiones con soluciones de agua regia a $80^{\circ} \mathrm{C}$, con agitación mecánica a $300 \mathrm{rpm}$ durante tres horas. Una vez frías las soluciones se filtró y se hicieron las diluciones necesarias para realizar la cuantificación de metales disueltos en un equipo de caracterización de absorción atómica Perkin Elmer 3110.

\subsection{Procedimiento experimental}

Muestras de $20 \mathrm{~g}$ de los diferentes tamaños de partícula fueron sometidas a disolución empleando soluciones de ácido nítrico, ácido sulfúrico y ácido clorhídrico. Estos ensayos se realizaron a temperatura ambiente $\left(18^{\circ} \mathrm{C}\right)$ en matraces de vidrio con agitación mecánica constante de $300 \mathrm{rpm}$. Alícuotas de las diferentes soluciones fueron tomadas a diversos tiempos para evaluar el grado de disolución de cobre. Los valores experimentales reportados, tanto en forma numérica y gráfica, corresponden a las medias aritméticas de los resultados obtenidos por triplicado a las condiciones específicas de operación.

\section{RESULTADOS Y ÁNALISIS}

Cada una de las muestras escogidas se sometió a la acción de agua regia, como se explicó anteriormente, para producir la disolución completa de los diferentes metales contenidos en ellas. Los elementos químicos

Rev. Ing. Investig. Desarro. Vol.18 (2). pp. 15-21, Julio-Diciembre. 2018, Sogamoso-Boyacá, Colombia. 
valorados, que son de interés para este trabajo, fueron cobre, oro y plata.

Tabla 1. Contenido de metales respecto al tamaño medio de partículas

\begin{tabular}{cccccc}
\hline Metales & $\begin{array}{c}\mathbf{1 6 5 1} \\
\boldsymbol{\mu m}\end{array}$ & $\begin{array}{c}\mathbf{- 1 6 5 1} \\
\mathbf{+ 8 3 3} \\
\boldsymbol{\mu m}\end{array}$ & $\begin{array}{c}\mathbf{- 8 3 3} \\
\mathbf{+ 4 9 0} \\
\boldsymbol{\mu m}\end{array}$ & $\begin{array}{c}\mathbf{- 4 9 0} \\
\mathbf{+ 2 4 6} \\
\boldsymbol{\mu m}\end{array}$ & $\begin{array}{c}\mathbf{- 2 4 6} \\
\mathbf{+ 1 7 3} \\
\boldsymbol{\mu m}\end{array}$ \\
\hline $\mathrm{Au}$ & & & & & \\
$\begin{array}{c}\mathrm{Ag} / \mathrm{t}) \\
\mathrm{Ag}\end{array}$ & 105 & 1269 & 131 & 59 & 60 \\
$\begin{array}{c}(\mathrm{g} / \mathrm{t}) \\
\mathrm{Cu} \\
(\mathrm{Kg} / \text { ton })\end{array}$ & 17567 & 2440 & 310 & 2274 & 180 \\
\hline
\end{tabular}

Como puede observarse en la tabla 1, la distribución de los metales no es homogénea en cada tamaño de partícula. Esto sucede por el comportamiento mecánico diferenciado de los distintos componentes de los residuos, que en algunos momentos pueden pulverizarse dada su fragilidad o laminarse según el carácter metálico que posean. Puede deberse también a la heterogeneidad en la composición de las tarjetas de reciclaje que se emplearon puesto que son de diferentes proveedores. [15] Resulta importante resaltar que esta etapa de preparación física podría emplearse como un método para preseleccionar los residuos de acuerdo con el metal que se considere de interés.

Las muestras fueron sometidas a tratamiento con las soluciones ácidas ya mencionadas para producir la disolución selectiva del cobre contenido en ellas. Los resultados de estas lixiviaciones pueden observarse a continuación en la figura 4.

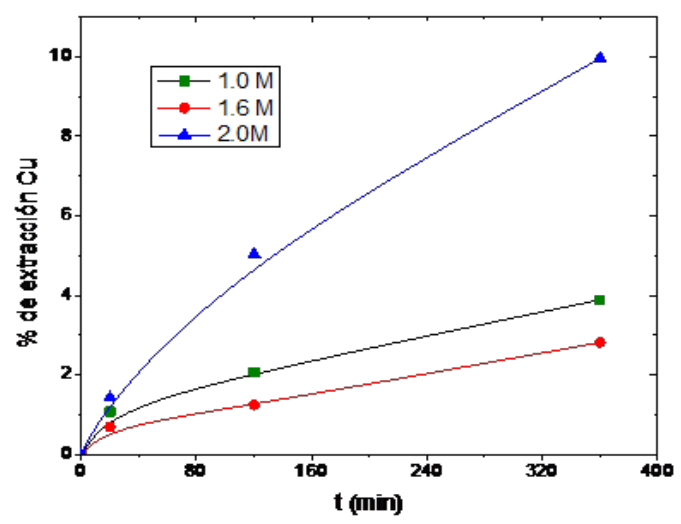

a)

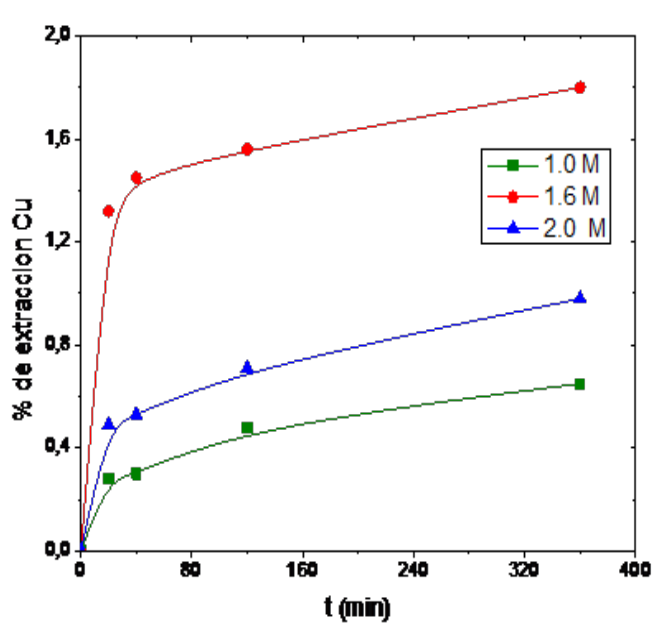

b)

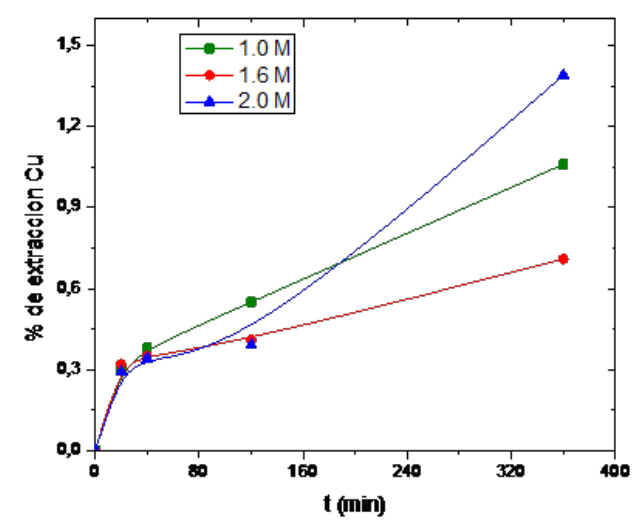

c)

Figura 4. Efecto de las concentraciones molares con ácidos inorgánicos desde tarjetas de computador PCB para la disolución de cobre. Todas las pruebas fueron realizadas con agitación mecánica de $300 \mathrm{rpm}$, temperatura ambiente $\left(18^{\circ} \mathrm{C}\right)$ y en un tiempo total de 360 minutos. (a) Proceso de lixiviación con $\mathrm{HNO}_{3}$, (b) Proceso de lixiviación con $\mathrm{HCl}$ (c) Proceso de lixiviación con $\mathrm{H}_{2} \mathrm{SO}_{4}$.

En las gráficas anteriores se indica que, en la mayoría de los procesos de extracción, se obtuvo un mayor porcentaje de disolución con las concentraciones más altas. Este efecto ocurre debido a que las tarjetas de computador contienen otro tipo de metales base como hierro, plomo, níquel y se disuelven a potenciales menores a $0.25 \mathrm{~V}$, según el diagrama de Pourbaix del cobre, figura 
1. Por esta razón la presencia de estos metales hace necesaria la utilización de concentraciones para los ácidos que estén por encima de la cantidad estequiométrica para la disolución del cobre y de esta manera alcanzar un mayor porcentaje de cobre disuelto.

El $\mathrm{HNO}_{3}$ es el reactivo con mayor porcentaje de extracción de cobre, en comparación a los otros dos reactivos $\mathrm{HCl}$ y $\mathrm{H}_{2} \mathrm{SO}_{4}$. Esto puede suceder por la capacidad de disolución del complejo formado entre el ácido nítrico y el cobre, lo cual se observa al analizar los logs $\mathrm{K}$, constante de disolución, de cada uno de los complejos formados con los otros reactivos, tabla 2, donde se muestra que el ácido nítrico tiene una alta capacidad de disolución con el cobre.

Tabla 2. Datos termodinámicos del proceso de lixiviación de cobre con ácidos inorgánicos [14].

\begin{tabular}{cc}
\hline REACCIÓN & Log K \\
\hline & \\
$2 \mathrm{Cu}+4 \mathrm{HNO}_{3}+\mathrm{O}_{2}=2 \mathrm{Cu}\left(\mathrm{NO}_{3}\right)_{2}+2 \mathrm{H}_{2} \mathrm{O}$ & 83.984 \\
$2 \mathrm{Cu}+2 \mathrm{H}_{2} \mathrm{SO}_{4}+\mathrm{O}_{2}=2 \mathrm{CuSO}_{4}+2 \mathrm{H}_{2} \mathrm{O}$ & 72.313 \\
$4 \mathrm{Cu}+4 \mathrm{HCl}+\mathrm{O}_{2}=4 \mathrm{CuCl}_{2}+2 \mathrm{H}_{2} \mathrm{O}$ & 76.375 \\
\hline
\end{tabular}

La mayoría de los resultados obtenidos en las lixiviaciones de cobre con los tres ácidos inorgánicos son relativamente bajos y no se logra obtener la extracción completa del metal. Este efecto ocurre debido a que metales como el cobre, se encuentran en forma aleada en los residuos electrónicos; algunos autores $[9,16-$ 17] sugieren que es necesario trabajar procesos de lixiviación oxidativa o utilizar mezclas de diferentes reactivos para la extracción completa de este metal.

Para mejorar el porcentaje de extracción del cobre, se realizó un proceso de lixiviación con ácido nítrico 2.0 $\mathrm{M}$ mezclado con peróxido de hidrógeno $0.5 \mathrm{M}$. Como se muestra en la figura 5, efectivamente si fueron más altos los porcentajes de extracción para el cobre en un tiempo de 400 minutos. Sin embargo, al observar la gráfica se indica que el proceso de lixiviación continúa, lo cual muestra que la reacción que controla el proceso es lenta y para obtener mayores resultados es necesario utilizar un tiempo superior a los 360 minutos.

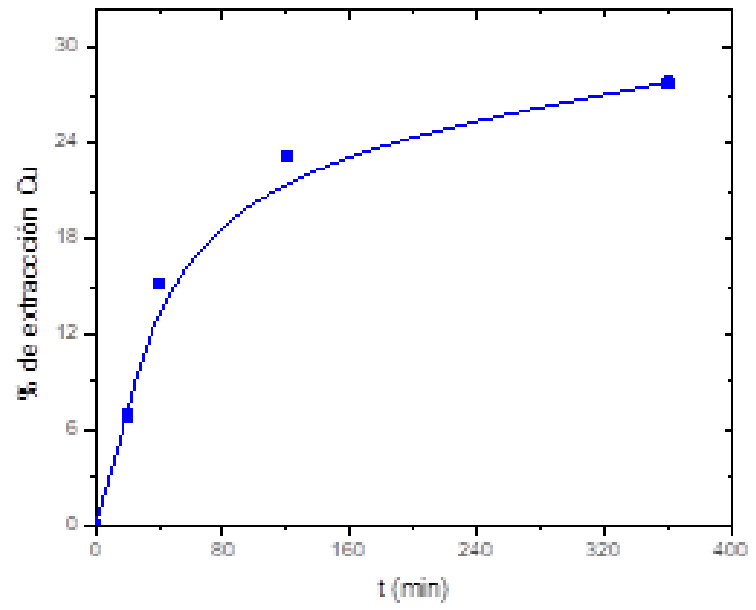

Figura 5. Proceso de lixiviación de cobre con $\mathrm{HNO}_{3} 2.0 \mathrm{M}$ y $\mathrm{H}_{2} \mathrm{O}_{2} 0.5 \mathrm{M}$. Se utilizó una relación sólido- líquido de 20 gramos por litro de solución, velocidad de agitación de $300 \mathrm{rpm}$ temperatura de $18^{\circ} \mathrm{C}$ y presión de $535 \mathrm{~mm} \mathrm{Hg}$.

Para valorar el efecto de la eliminación de cobre y otros elementos metálicos no cuantificados en este trabajo, pero que, si están contenidos en los residuos ensayados, se procedió a analizar los sólidos lixiviados de los procesos de disolución ejecutados. El análisis de este proceso se presenta en la figura 6.

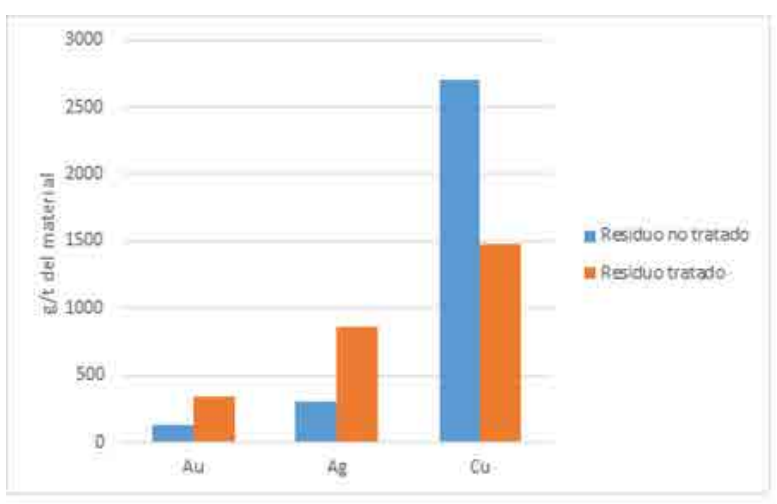

Figura 6. Comparación de contenidos metálicos de interés en residuos tratados y no tratados

Este material procesado, resulta apropiado para utilizarlo como materia prima en la recuperación de oro y plata de los residuos PCB, puesto que los elementos consumidores de reactivos empleados en la disolución de metales preciosos, especialmente el cobre, se han reducido de manera notoria. 
Para comprobar los resultados obtenidos de la eliminación del cobre, se realizó el proceso de extracción de oro y plata con soluciones de tiourea. En la figura 7 se observa que es más efectivo el proceso de la eliminación del cobre para la posterior recuperación de metales preciosos como oro y plata.

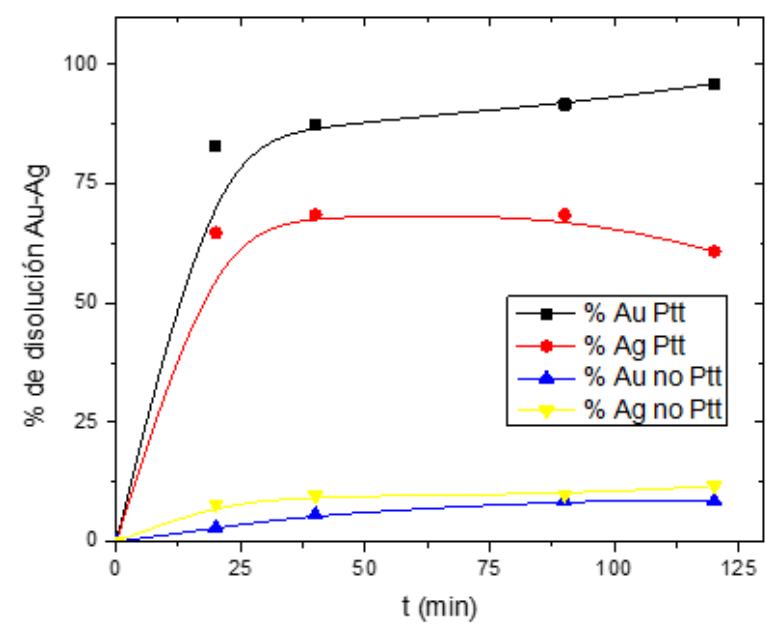

Figura 7. Proceso de lixiviación de oro y plata con Tiourea 0.2 $\mathrm{M}$ activada con $\mathrm{H}_{2} \mathrm{O}_{2}$. Se utilizó una relación sólido- líquido de 200 gramos por litro de solución, velocidad de agitación de $300 \mathrm{rpm}$ temperatura de $18^{\circ} \mathrm{C}$ y presión de $535 \mathrm{~mm} \mathrm{Hg}, \mathrm{pH}$ 1.5. a) Proceso de lixiviación sin limpieza de cobre. (No Ptt ) y con limpieza de cobre (Ptt).

\section{CONCLUSIONES}

El cobre es el metal que se encuentra en mayor proporción en las tarjetas electrónicas PCB con un $27.09 \%$. El oro y la plata se encuentran en proporciones con $131 \mathrm{~g} /$ ton y $310 \mathrm{~g} /$ ton respectivamente.

Además del cobre, los ácidos inorgánicos también disuelven otros elementos metálicos contenidos en los residuos como hierro, níquel, plomo, zinc, etc. generando en consecuencia un aumento en la concentración de oro desde $131,29 \mathrm{~g} /$ ton a $345,9 \mathrm{~g} /$ ton y en la plata desde $310 \mathrm{~g} /$ ton a $864,8 \mathrm{~g} /$ ton.

Este material procesado resulta muy adecuado para intentar la posterior recuperación de oro y plata, como se demostró en el proceso de lixiviación de residuos con soluciones de tiourea. En el proceso de lixiviación sin extracción de cobre, se consigue el $11.71 \%$ de recuperación de plata y $8.63 \%$ para oro, mientras que, en la lixiviación de residuos con extracción de cobre, la recuperación aumenta a $60.9 \%$ para plata y $96.06 \%$ para oro.

\section{AGRADECIMIENTOS}

Este trabajo de investigación se desarrolló en el grupo de investigación de Metalurgia No Ferrosa de la Universidad Pedagógica y Tecnológica de Colombia, Tunja, Boyacá. Se agradece a la Dirección de Investigaciones de la UPTC por los recursos económicos aportados para su realización.

\section{REFERENCIAS}

[1] R. Cayumil, R. Khanna, R. Rajarao, P.S. Mukherjee, V. Sahajwalla, "Concentration of precious metals during their recovery from electronic waste", Waste Management, vol. 57, pp 121-130, 2016. DOI: http://dx.doi.org/10.1016/j.wasman.2015.12.004

[2] Este año Colombia tendrá 143.000 toneladas de residuos electrónicos. [En línea]. El Tiempo. Bogotá D.C. 28, agosto, 2015. [Consulta: enero 2015]. Mensaje archivado en http://www.eltiempo.com/ archivo/documento/CMS-13156235.

[3] El planeta tiro a la basura 180 billones de pesos. [En línea]. El Espectador. Bogotá D.C. 14, diciembre, 2017. [Consulta: mayo 2018]. Mensaje archivado en https://www.elespectador.com/noticias/ medio-ambiente/el-planeta-tiro-la-basura-180-billones-de-pesos-articulo-728484.

[4] Y. Reyes, I. Vergara, O. Torres, M. Diaz-Lagos, E.E. González-Jimenez, "Contaminación por metales pesados: Implicaciones en salud, ambiente y seguridad alimentaria", Revista Ingeniería Investigación y Desarrollo, vol. 16 (2), pp. 66-77, julio, 2016. DOI: $\quad$ https://doi.org/10.19053/1900771X.v16. n2.2016.5447

[5] J. Huang, M. Chen, H. Chen, S. Chen, Q. Sun, "Leaching behavior of copper from waste printed circuit boards with Brønsted acidic ionic liquid", Waste Management, vol. 34, pp. 483-388, 2014. DOI:http://dx.doi.org/10.1016/j.wasman.2013.10.027.

[6] S. Yousef, M. Tatariants, R. Bendikiene, G. Denafas,"Mechanical and thermal characterizations of non-metallic components recycled from waste printed circuit boards", Journal of Cleaner Production, vol. 167, pp. 271-280, 2017. DOI: http://dx. doi.org/10.1016/j.jclepro.2017.08.195. 
[7] A. Kumar, M. Holuszko, T. Janke, "Characterization of the non-metal fraction of the processed waste printed circuit boards", Waste Management, vol. 75, pp. 94-108, 2018. DOI: https://doi.org/10.1016/j.wasman.2018.02.010.

[8] L. Diaz, T. Lister, "Economic evaluation of an electrochemical process for the recovery of metals from electronic waste", Waste Management, vol. 74, pp. 384-392, 2018. DOl:https://doi.org/10.1016/j. wasman.2017.11.050

[9] H. Lee, B. Mishra, "Selective recovery and separation of copper and iron from fine materials of electronic waste processing", Minerals Engineering, vol. 123, pp. 1-7, 2018. DOI: https://doi.org/10.1016/j.mineng.2018.04.021

[10] S. Fogarasi, F. Imre-lucaci, Á. Imre-lucaci, P. Ilea,"Copper recovery and gold enrichment from waste printed circuit boards by mediated electrochemical oxidations", Journal of Hazardous Materials, pp 215-221, 2014. DOI: http://dx.doi.org/10.1016/j. jhazmat.2014.03.043.

[11] Y. Lu, Z. Xu, "Precious metals recovery from waste printed circuit boards: A review for current status and perspective", Resources, Conservation and Recycling, vol. 113, pp. 28-39, 2016. DOI: http://dx. doi.org/10.1016/j.resconrec.2016.05.007.
[12] M. Parra. "Los diagramas de Pourbaix" en Universidad Pedagógica y Tecnológica de Colombia, 1989, pp. 1-4.

[13] NIST, 2004. Critically Selected Stability Constants of Metal Complexes. NIST Standard Reference Database 46, Version 8.0.

[14] Robinson Torres, Gretchen T. Lapidus. "Copper leaching from electronic waste for the improvement of gold recycling", Waste Management, vol. 57, November 2016. pp 131 - 139. DOI:http://dx.doi. org/10.1016/j.wasman.2016.03.010

[15] Base de datos plataforma HSC CHEMISTRY 5.11 for Windows.

[16] I. Birloaga, I. Michelis, F. Ferella, M. Buzatu, F. Veglio, "Study on the influence of various factors in the hydrometallurgical processing of waste printed circuit boards for copper and gold recovery", Waste Management, vol. pp 935-941, 2013. DOI: http://dx.doi.org/10.1016/j.wasman.2013.01.003

[17] E.Y. Yacizi, H. Deveci, "Ferric sulphate leaching of metals from waste printed circuit boards", International Journal of Mineral Processing, vol. 133, pp. 39-45,2014. DOI: http://dx.doi.org/10.1016/j. minpro.2014.09.015. 